\title{
Multi-scale Colorectal Tumour Segmentation Using a Novel Coarse to Fine Strategy
}

Kun Zhang ${ }^{1,2}$

Danny Crookes ${ }^{3}$

Jim Diamond ${ }^{4}$

Minrui Fei ${ }^{1}$

Jianguo $\mathrm{Wu}^{2}$

\author{
Peijian Zhang ${ }^{2}$ \\ Huiyu Zhou ${ }^{3}$ \\ ${ }^{1}$ Shanghai University, China \\ ${ }^{2}$ Nantong University, China \\ ${ }^{3}$ Queen's University Belfast, UK \\ ${ }^{4}$ PathXL Ltd., Belfast, UK
}

Colorectal cancer is the third most common form of cancer worldwide [1]. In general, there are two challenges in automatic detection of colorectal cancer in histopathological images. One is the enormous volume of data which the algorithms have to cope with. The second is that in histopathology slides, cancerous tissue can look similar to noncancerous tissue.

In this paper, we propose an efficient and novel coarse to fine framework to address the problem of colorectal tumour segmentation for the purpose of tumour detection, as shown in Fig 1. In this way, we propose the use of colour modelling and morphological operations to extract the initial Region of interest (ROI). In order to reduce the noisy margin, we use Euclidean distance based histograms as a criterion. Further, to perform tumour segmentation at the best resolution, we deploy a Convergence Index (CI) approach to detect nuclei by fitting circles. At the tumour classification stage, when the resolution is at a high level, we use a rotation invariant feature and random projection based $1_{2}$-norm sparse representation technique for more accurate segmentation. The main contributions of our work include: 1) a multi-scale strategy for tumour segmentation, which simulates the decision making of a pathologist from the coarse-to-fine processing, and 2) a novel Rotation Invariant Raw Statistics (RIRS) feature and random projection based $1_{2}$-norm sparse representation method. These make the tumour classification process more effective than other published methods.

The RIRS-pixel feature can be calculated by: $x^{p i x e l}=\left[x_{0,0},\left(x_{1,1}, x_{1,2}, x_{1,3}, x_{1,4}\right), \ldots,\left(x_{8,1}, x_{8,2}, x_{8,3}, x_{8,4}\right)\right]^{T}$ where $x_{i, j}$ is a pixel with different scales and orientations. $i$ is scale and $j$ is orientation.

The new texton learning model is formed by: $\min _{D, \alpha}\left(\|x-D K\|_{2}^{2}+\lambda\|K\|_{2}^{2}+\eta \sum_{i=1}^{n}\left\|\alpha_{i}-\delta\right\|_{2}^{2}\right)$ s.t $d_{j}^{T} d_{j}=1$

where $D=\left[d_{1}, d_{2}, \ldots, d_{j}\right], d_{k} \in R^{m}$ is the texton learning model. $K=\left[\alpha_{1}, \alpha_{2}, \ldots, \alpha_{l}\right]$ are coding coefficients. $\delta$ is the mean of all $\alpha_{i}$ which may vary by $50 \%$ more or less than the mean value. $\delta=1 / n \sum_{i=1}^{n} \alpha_{i}$ when distortion does not appear. When distortion appears, $\delta=1 /(n-u)\left(\sum_{i=1}^{n} \alpha_{i}\right.$ $-\sum_{f=1}^{u} \alpha_{f}$ ), where $u$ is the distortion.
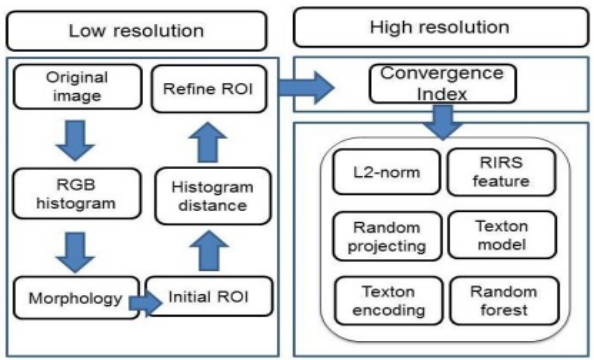

Figure 1: Overview of the proposed segmentation algorithm.

A total of $20 \mathrm{H} \& \mathrm{E}$ stained colorectal cancer slides were supplied by a medical imaging company and used as the basis for training and testing. We compare our method against recently published texture classification methods (ELBP, MR8, TEISF, Patch, and SRP). The results favor the proposed approach in terms of classification accuracy.

[1] J. Ferlay, et al., Cancer incidence and mortality worldwide. International Agency for Research on Cancer, 2010. 